Bundesgesundheitsbl 2020 - 63:1241-1249 https://doi.org/10.1007/s00103-020-03217-5 Eingegangen: 14. November 2019 Angenommen: 22. August 2020 Online publiziert: 22. September 2020 (c) Der/die Autor(en) 2020

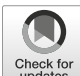

Claudia Maria Schmidt ${ }^{1} \cdot$ Saskia Klein ${ }^{2} \cdot$ Philippe Stock $^{3} \cdot$ Konrad Reinshagen ${ }^{2,4}$. Ingo Königs s,4

'Interdisziplinärer Kinderschutz, Altonaer Kinderkrankenhaus, Hamburg, Deutschland

${ }^{2}$ Abteilung für Kinderchirurgie, Altonaer Kinderkrankenhaus, Hamburg, Deutschland

${ }^{3}$ Abteilung für Pädiatrie, Altonaer Kinderkrankenhaus, Hamburg, Deutschland

${ }^{4}$ Klinik und Poliklinik für Kinderchirurgie, Universitätsklinikum Hamburg-Eppendorf, Hamburg,

Deutschland

\title{
Kindeswohlgefährdung - Ausweitung des Hellfeldes durch Neukonzeption der klinischen Kinderschutzarbeit
}

In offiziellen Statistiken wird die Prävalenz für Kindeswohlgefährdung in Deutschland aufgrund einer hohen Dunkelziffer erheblich unterschätzt [5]. Im Rahmen einer Querschnittsuntersuchung einer repräsentativen Stichprobe der deutschen Gesamtbevölkerung berichten $12 \%$ der Befragten von körperlicher Misshandlung, 14,9\% von emotionaler Misshandlung, 10,8\% von schwerer körperlicher und 6,6\% von schwerer emotionaler Vernachlässigung sowie $12,5 \%$ von sexuellem Missbrauch [6]. Diese retrospektiv berichteten Häufigkeiten entsprechen dabei teils den Ergebnissen internationaler Studien [7-10]. Körperliche Misshandlung (14,3-54,8\%), emotionale Misshandlung (11,3-46,7\%) und emotionale Vernachlässigung (14,5-40,0\%) werden im internationalen Vergleich teils vermehrt angegeben [7].

Kindeswohlgefährdung ist mit beträchtlichen negativen Konsequenzen [11] wie Beeinträchtigungen der physischen $[12,13]$ und psychischen Gesundheit [14, 15], der kognitiven Entwicklung [16-18] sowie Störungen des Sozialverhaltens [19-22] assoziiert. Darüber hinaus entstehen immense gesellschaftliche Folgekosten [23]. Insofern ist bei Verdacht auf Kindeswohlgefährdung eine frühzeitige Intervention von zentraler Bedeutung, sowohl hinsichtlich der Verbesserung der Entwicklungschancen der
Kinder und Jugendlichen und deren späterer Teilhabe am gesellschaftlichen Leben als auch aus nüchternen ökonomischen Gründen.

Mit dem Inkrafttreten des Bundeskinderschutzgesetzes (BKiSchG) im Jahr 2012 wurde neben einer weiteren gesetzlichen Grundlage für die Durchbrechung der (ärztlichen) Schweigepflicht die Basis für eine interdisziplinäre Zusammenarbeit im Kinderschutz geschaffen. Verantwortungsträger der Kinder- und Jugendhilfe sowie des Gesundheitswesens werden ausdrücklich zur gemeinsamen Kooperation verpflichtet. Erfolgreiche Prävention bzw. Intervention bei Kindeswohlgefährdung soll als systemübergreifende Aufgabe begriffen und angegangen werden.

Für Kinderkliniken werden spätestens seit der Einführung des mittlerweile erlöswirksamen Operationen- und Prozedurenschlüssels OPS 1-945 (Diagnostik bei Verdacht auf Gefährdung von Kindeswohl und Kindergesundheit) und der Erfassung von Kindeswohlgefährdung durch den Code T74 in der Internationalen Klassifikation der Krankheiten (ICD) die Installation von strukturierten Verfahren sowie die Etablierung von interdisziplinären Kinderschutzgruppen gefordert [24]. Die Umsetzung der damit verbundenen Leistungsanforderungen, die in der aktuell veröffentlichten S3Leitlinie „Kindesmisshandlung, -miss- 


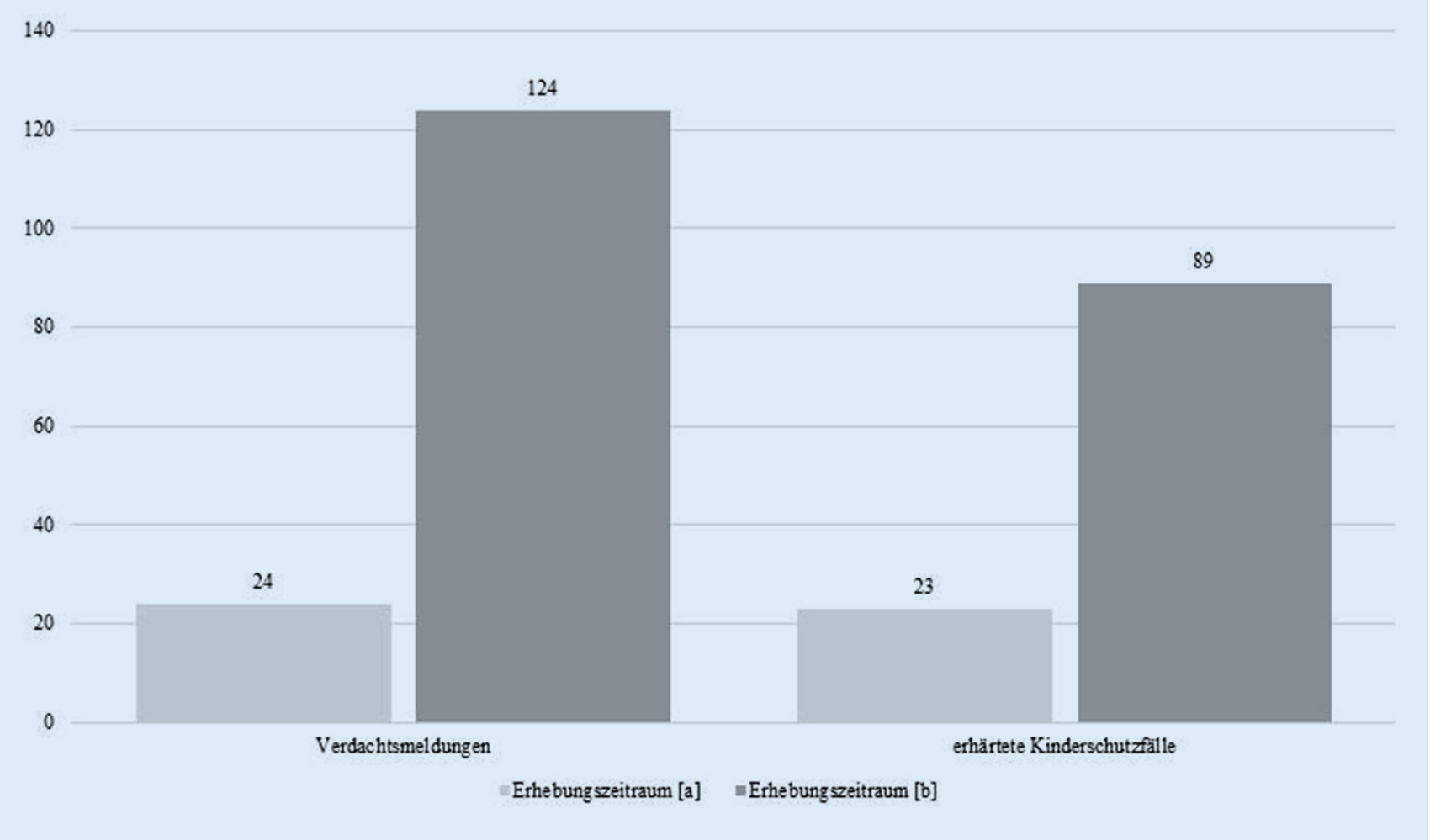

Abb. 1 \ Verdachtsmeldungen $(n=148)$ und erhärtete Kinderschutzfälle $(n=112)$ der Erhebungszeiträume [a] und [b]

brauch, -vernachlässigung unter Einbindung der Jugendhilfe und Pädagogik“ umfassend beschrieben sind [25], stellt im klinischen Alltag aufgrund fehlender Kapazitäten sowie entsprechender Kompetenzen oftmals eine große Herausforderung dar.

Vor diesem Hintergrund wurde am Altonaer Kinderkrankenhaus, einer großen Klinik der gesundheitlichen Regelversorgung in Hamburg, im Rahmen eines zunächst spendenfinanzierten Modellprojekts eine hauptamtliche Kinderschutzkoordinatorin implementiert. Ausgestattet mit umfangreichen zeitlichen Ressourcen sowie spezifischen Qualifikationen wurde innerhalb dieser Funktion der Vorsitz der bereits bestehenden Kinderschutzgruppe übernommen sowie die Weiterentwicklung der klinikinternen Kinderschutzarbeit in Form von Sensibilisierung, Fortbildungen, konzeptioneller und struktureller Neuorganisation vorangetrieben.

\section{Methode}

Im Rahmen einer retrospektiven Querschnittstudie wurde die Entwicklung der am Altonaer Kinderkrankenhaus registrierten (Verdachts-)Fälle von Kindeswohlgefährdung im Zusammenhang mit der Implementierung der hauptamtlichen Kinderschutzkoordinatorin evaluiert. Untersucht wurden alle erfassten (Verdachts-)Fälle der jeweils 24-monatigen Vergleichszeiträume [a] vor der Tätigkeit der Kinderschutzkoordinatorin (01.04.2013-31.03.2015) sowie [b] während ihrer Tätigkeit (01.04.2015-31.03. 2017). Es wurden neben der Art der Kindeswohlgefährdung und epidemiologischen Daten die Art und Lokalisation der Verletzungen sowie anamnestische Hinweise zur Diagnosefindung der Kindeswohlgefährdung erfasst. Die Datenerhebung erfolgte anhand der elektronischen Patientenakten. Die statistische Auswertung erfolgte mittels SPSS Statistics 25.0. Die erhobenen Daten beider Zeiträume wurden miteinander verglichen. Zur Überprüfung von Unterschieden wurden $\mathrm{Chi}^{2}$-Tests sowie Exakte Fisher-Tests durchgeführt. Als signifikant galt ein $p$-Wert $<0,05$. Die Unterschiede wurden, wenn nicht anders ausgewiesen, auf die Anteile der jeweiligen Populationen errechnet.

\section{Ergebnisse}

Im Erhebungszeitraum [a] wurden am Altonaer Kinderkrankenhaus $N=84.815$ Kinder und Jugendliche im Rahmen stationärer, teilstationärer und ambulanter medizinischer Versorgung behandelt. Bei $n=24$ Patienten wurde unter Beteiligung der klinikinternen Kinderschutzgruppe dem Verdacht einer Kindeswohlgefährdung nachgegangen. In der überwiegenden Mehrheit der Fälle $(95,8 \%)$ hat sich im Rahmen des Abklärungsprozesses der Verdacht auf Kindeswohlgefährdung erhärtet (• Abb. 1) und damit weitere Maßnahmen, wie z. B. die Hinzuziehung des Jugendamts, erforderlich gemacht. Die $n=23$ erhärteten Kinderschutzfälle zeigten sich in Form von Misshandlung $(52,2 \%)$ und Vernachlässigung $(47,8 \%$; - Abb. 2). Die Altersverteilung ist in - Abb. 3 dargestellt. Die überwiegen- 
Bundesgesundheitsbl 2020 • 63:1241-1249 https://doi.org/10.1007/s00103-020-03217-5

(c) Der/die Autor(en) 2020

\section{M. Schmidt · S. Klein · P. Stock $\cdot$ K. Reinshagen $\cdot$ I. Königs}

\section{Kindeswohlgefährdung - Ausweitung des Hellfeldes durch Neukonzeption der klinischen Kinderschutzarbeit}

\section{Zusammenfassung}

Hintergrund. Das Erkennen von Kindesmisshandlungen und der Umgang damit sind Herausforderungen im ärztlichen Alltag. Die Dunkelziffer bei Misshandlungen und Vernachlässigungen ist hoch.

Ziel der Arbeit. Das Ziel der Arbeit war, die Effektivität des neu strukturierten Kinderschutzprogrammes einer großen deutschen Kinderklinik zu untersuchen und durch die Präsentation der Vorteile der neuen Struktur die Verbesserung von Programmen für Kinder und Mediziner in anderen Kliniken anzuregen.

Methode. Es wurden retrospektiv alle Kinderschutzfälle im Altonaer Kinderkrankenhaus vor und nach Neukonzeption der Kinderschutzarbeit über einen Zeitraum von jeweils 2 Jahren untersucht und miteinander verglichen. Die Kinderschutzgruppe war neu strukturiert worden und wurde nachfolgend von einer hauptamtlichen Kinderschutzkoordinatorin geleitet.

Ergebnis. Die Prävalenz der Verdachtsfälle und der erhärteten Fälle von Kindeswohlgefährdung ist im Untersuchungszeitraum nach Neukonzeption hoch signifikant erhöht. Im Zeitraum vor der Neukonzeption wurden 24 Fälle mit dem Verdacht auf Kindswohlgefährdung untersucht, 23 erhärtet, danach 124 Fälle untersucht, 89 erhärtet. Eine Stigmatisierung der Familien bei der Abklärung wurde trotz des signifikanten Anstiegs falsch-positiver Verdachtsfälle durch sensibles Vorgehen und eine Konfrontation erst bei Erhärtung des Verdachtes vermieden. Schlussfolgerung. Das vorgestellte Konzept zum medizinischen Kinderschutz in Kinderkliniken scheint das Hellfeld bei Kindeswohlgefährdung effektiv auszuweiten. Sowohl die Reduktion nichtdetektierter Fälle mit der Verhinderung potenzieller Eskalation mit prolongiertem Leidensweg als auch die Verminderung der immensen gesellschaftlichen Folgekosten rechtfertigen die personellen Investitionen.

Schlüsselwörter

Kindermisshandlung $\cdot$ Kinderschutz .

Vernachlässigung · Dunkelziffer · Kindeswohlgefährdung

\section{Child abuse - reduction in the estimated number of unreported cases by restructuring a clinical child protection program}

Abstract

Background. Identification of child abuse is a daily challenge in medical work. The estimated number of unreported cases of child abuse and neglect is high.

Objectives. The aim of this study was to investigate the effectiveness of the redesigned clinical child protection program of a major German pediatric hospital and to improve programs in other hospitals for children and physicians through presentation of the advantages of the new structure.

Methods. All cases of child protection at the Altona Children's Hospital were retrospectively analyzed before and after restructuring of the clinic's child protection program for a two-year period each, and a comparison was made. The child protection program was restructured and the new program subsequently managed by a fulltime coordinator.

Results. The prevalence of both suspected and substantiated cases of child abuse was significantly higher after restructuring of the child protection program. Before the change, 24 cases were investigated, of which 23 were substantiated; afterward, 124 cases were investigated and 89 were substantiated. Despite the high number of false-positive suspected cases, stigmatization of the families during the clarification progress was avoided by using a very sensitive approach, and the family was not confronted until the suspicion was affirmed.

Conclusion. The presented concept of child protection in medical clinics seems to facilitate a higher detection rate of child abuse cases. The reduction in the number of undetected cases and thereby prevention of possible escalation of abuse, as well as the decline in the immense social follow-up costs, justifies the increased personnel costs.

Keywords

Child abuse - Child protection - Neglect . Estimated number of unreported cases . Child hazard de Mehrheit (65,2\%) war männlichen Geschlechts.

Im Erhebungszeitraum [b] wurden $N=106.289$ Kinder und Jugendliche medizinisch versorgt. Unter der Leitung der hauptamtlichen Kinderschutzkoordinatorin wurde die klinikinterne Kinderschutzgruppe bei $n=124$ Patienten zur Abklärung des Verdachts auf Kindeswohlgefährdung hinzugezogen. Das Einleiten weiterer Maßnahmen war bei $n=89$ Kindern und Jugendlichen $(71,8 \%)$ aufgrund des erhärteten Ver- dachts (• Abb. 1) auf Vernachlässigung (51,7\%), Misshandlung (41,6\%) und sexuellen Missbrauch (6,7\%) erforderlich (-Abb. 2). Die Altersverteilung ist in - Abb. 3 dargestellt. Die Hälfte war männlichen Geschlechts (53,9\%).

Die Prävalenz der Verdachtsfälle ist im Erhebungszeitraum [b], also seit Implementierung der hauptamtlichen Kinderschutzkoordinatorin signifikant $(p<0,000)$ gestiegen. Auch die Prävalenz der Fälle, bei denen sich im Rahmen des Abklärungsprozesses der Verdacht auf Kindeswohlgefährdung erhärtet hat, zeigt einen signifikanten Anstieg $(p<0,000$; Abb. 1). Ein weiterer signifikanter Anstieg $(p<0,000)$ ist bei den falsch-positiven Verdachtsfällen festgestellt worden. Die Prävalenz der Erscheinungsformen Misshandlung und Vernachlässigung zeigte keine signifikanten Unterschiede in den Erhebungszeiträumen. Fälle von sexuellem Missbrauch wurden ausschließlich im Erhebungszeitraum [b] identifiziert (• Abb. 2). Der Anteil der Kinderschutzfälle mit 


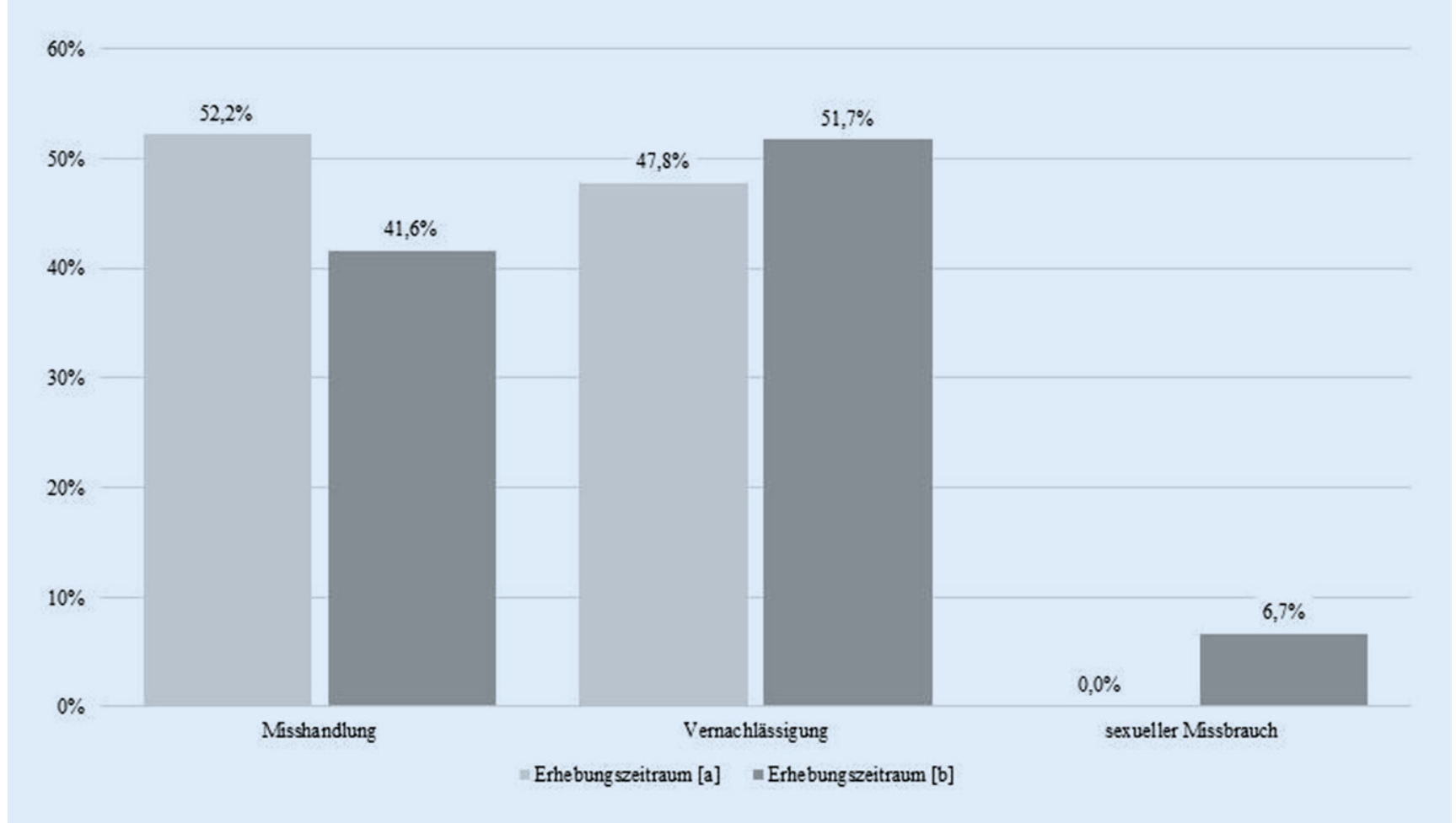

Abb. $2 \Delta$ Erscheinungsform der erhärteten Kinderschutzfälle $(n=112)$ der Erhebungszeiträume [a] und [b]

lebensbedrohlichen Verletzungen oder Mangelerscheinungen $(p<0,008)$ sowie die Prävalenz der Inobhutnahmen durch das jeweils zuständige Jugendamt $(p<0,031)$ ist im zeitlichen Verlauf von Zeitraum [a] zu [b] signifikant gesunken (- Abb. 4). Diagnostische und anamnestische Auffälligkeiten, die zum Verdacht der Kindeswohlgefährdung führten, sind in - Tab. 1 aufgeführt. Die Lokalisation der körperlichen Verletzungen unterschied sich in beiden Zeiträumen nicht signifikant. Die Kopf-Hals-Region war in allen Fällen zu 57,1\% am häufigsten betroffen, gefolgt von Thorax (44,9\%), unterer $(22,5 \%)$ und oberer (16,5\%) Extremität sowie dem Abdomen (10,2\%). Das Gesäß und das Genitale waren zu je 2,0 \% verletzt. Die Art der körperlichen Verletzungen unterschied sich ebenfalls nicht signifikant. Insgesamt lagen in $55,0 \%$ der Fälle Weichteilverletzungen, in $24,5 \%$ Frakturen und in $2,0 \%$ der Fälle thermische Verletzungen vor. Organverletzungen traten in $18,4 \% \mathrm{der}$ Fälle auf.

\section{Diskussion}

In den vergangenen Jahren wurde die Problematik von Kindeswohlgefährdungen vermehrt gesellschaftlich, politisch und medizinisch wahrgenommen und strukturelle Veränderungen wurden eingeleitet. Meilensteine dieser Entwicklung waren die Gründung der Deutschen Gesellschaft für Kinderschutz in der Medizin (DGKiM, ehemals Arbeitsgemeinschaft Kinderschutz in der Medizin) 2008, das Inkrafttreten des Bundeskinderschutzgesetzes 2012 und die Veröffentlichung der S3-Leitlinie Kinderschutz 2019. Einerseits konnte so die öffentliche Wahrnehmung gesteigert werden, andererseits wurden insbesondere unter Führung der DGKiM zahlreiche Fortbildungen und Zertifizierungslehrgänge durchgeführt, Standards definiert und Handlungsempfehlungen erstellt [24]. Neben zahlreichen nationalen und internationalen Publikationen wurde unter der Leitung der Klinik für Kinder- und Jugendpsychiatrie/ Psychotherapie des Universitätsklinikums Ulm das webbasierte Weiterbildungsangebot E-Learning Kinderschutz etabliert sowie die medizinische Kinderschutzhotline (0800 $19210 \quad 00)$ eingerichtet [26, 27].

Im Altonaer Kinderkrankenhaus erfolgte die Kinderschutzarbeit seit 2008 durch eine interdisziplinäre Kinderschutzgruppe. Diese setzte sich, analog der „Empfehlungen für Kinderschutz an Kliniken“, aus Ärzten (Pädiatrie, Kinderchirurgie, Kinder- und Jugendpsychosomatik, Kinderradiologie, Rechtsmedizin), Mitarbeitern der psychosozialen Dienste sowie Pflegekräften zusammen [24]. Vor der Neustrukturierung wurde die Kinderschutzgruppe durch einen Oberarzt der Kinderklinik nebenamtlich geleitet. Im Jahr 2015 wurde die bereits bestehende Kinderschutzgruppe durch eine hauptamtliche, im interdisziplinären Kinderschutz spezialisierte Kinderschutzkoordinatorin ergänzt und fortan durch diese geleitet. Die Zusammensetzung der Kinderschutzgruppe wurde darüber hinaus nicht verändert, auch nicht der essenzielle interdisziplinäre Entscheidungsprozess. Im Falle eines Verdachts auf Kindeswohlgefährdung wird nun umgehend die Kinderschutzkoordinatorin infor- 


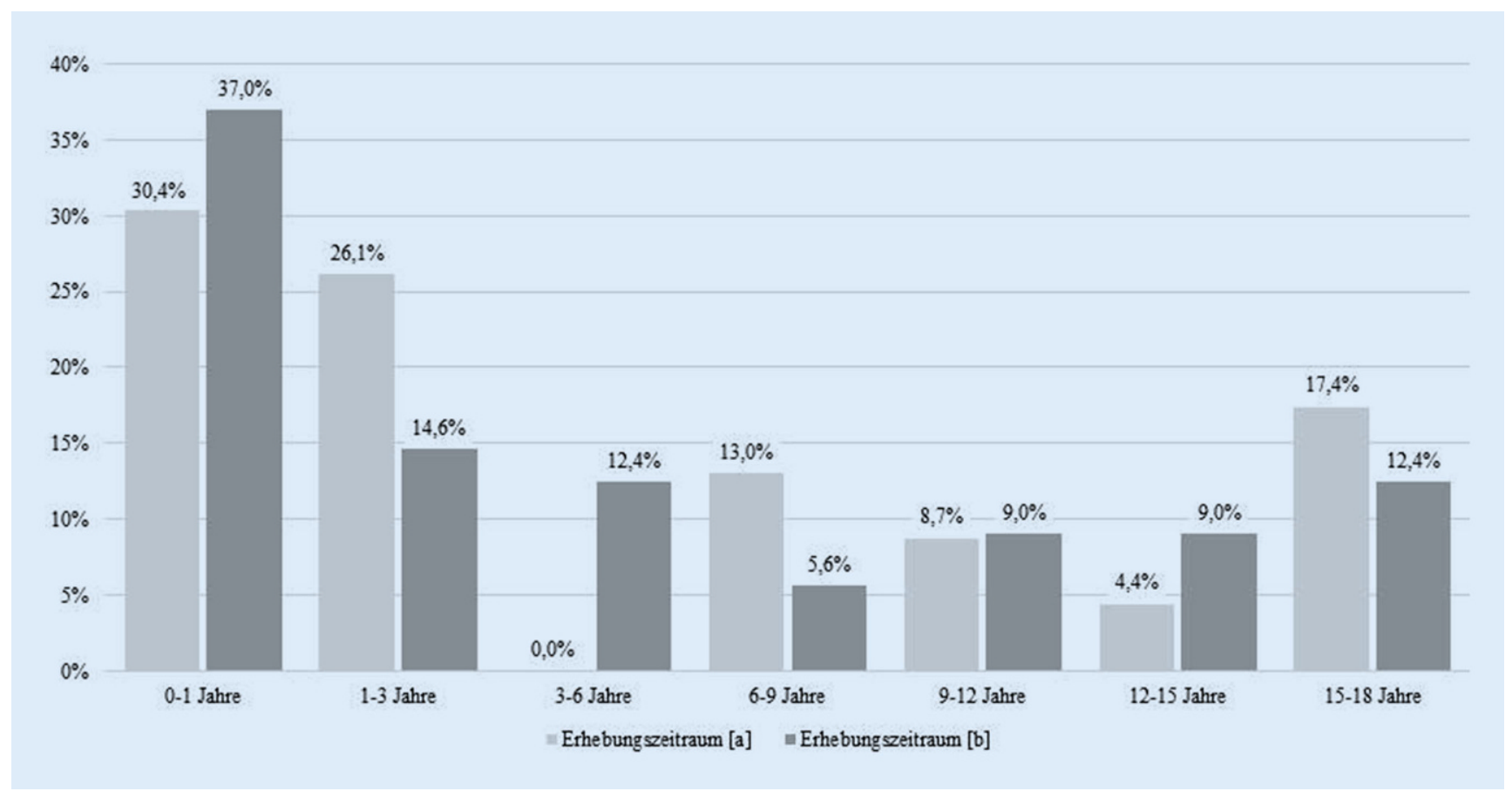

Abb. $3 \Delta$ Altersverteilung der erhärteten Kinderschutzfälle $(n=112)$ der Erhebungszeiträume [a] und [b]

miert und spätestens am Folgetag die interdisziplinäre Kinderschutzgruppe einberufen. Die gesamte Fallsteuerung und Kommunikation obliegen der Kinderschutzkoordinatorin. Aufgrund der erhöhten personellen Kapazität konnten Verdachtsfälle stringenter verfolgt und Abklärungsprozesse weiter strukturiert werden. Mit der Implementierung dieser personellen Ressourcen, deren exklusive Aufgabe neben der Fallarbeit die Weiterentwicklung des Kinderschutzes ist, wurde der Kinderschutz zunehmend als zentraler Bestandteil einer Kinderklinik wahrgenommen und akzeptiert sowie die Erreichbarkeit und Verfügbarkeit im klinischen Alltag gesteigert. Darüber hinaus wurden Meldewege deutlich vereinfacht und die Vernetzung von allen am Kinderschutz beteiligten Personen und Institutionen, wie z. B. andere (Kinder-)Kliniken, niedergelassene (Kinder-)Ärzte, Frühe Hilfen, Jugendund Gesundheitsämter, Kindertagesstätten und Schulen, aber auch Polizei, Staatsanwaltschaft und (Familien-)Gerichte, verbessert.

Nach Wissen der Autoren ist eine hauptamtliche Kinderschutzkoordination als Leitung der Kinderschutzgruppen in wenigen Kinderkliniken vorhanden.
Die Kinderschutzgruppen werden in der Regel nebenamtlich, durch ansonsten anderweitig klinisch beschäftige Personen geleitet, analog der Zusammensetzung im Altonaer Kinderkrankenhaus vor der Implementierung der Kinderschutzkoordinatorin.

Das Altonaer Kinderkrankenhaus ist eine der größten Kinderkliniken in Deutschland und repräsentiert aufgrund eines breiten und ausgewogenen Spektrums die Kinder- und Jugendmedizin optimal. Auch die Altersverteilung und die leichte Knabenwendigkeit dieser Untersuchung spiegelt die übliche Verteilung in der Literatur wider [28].

Die Untersuchung zeigt, dass nach der Neustrukturierung der Kinderschutzgruppe die Prävalenz der Verdachtsfälle als auch die Prävalenz der Fälle, bei denen sich der Verdacht auf Kindeswohlgefährdung erhärtet hat, signifikant gestiegen ist. Die verbesserte Koordination sowie die Weiterentwicklung der Kinderschutzarbeit, die mit der Implementierung der hauptamtlichen Kinderschutzkoordinatorin sowohl personell als auch strukturell erfolgte, scheinen die Qualität, die Effizienz und die Arbeitsweise der Kinderschutzgruppe deutlich zu verbessern.
Bei gleichbleibendem und in den untersuchten Zeiträumen nur numerisch größerem Patientenkollektiv ist wahrscheinlich weniger von einem tatsächlichen Anstieg der Prävalenz von Kindeswohlgefährdung als vielmehr von einer Verschiebung der Fälle vom Dunkelfeld ins Hellfeld auszugehen. Insofern ist denkbar, dass in der Zeit vor der Neustrukturierung und der damit verbundenen Weiterentwicklung der klinikinternen Kinderschutzarbeit Fälle von Kindeswohlgefährdung nicht erkannt und/oder verfolgt wurden. Mögliche Ursachen hierfür sind in internationalen Studien identifiziert worden: Wissenslücken und fachliche Unsicherheiten [29-32], Angst vor falschen Verdachtsäußerungen [30], Befürchtungen vor unangenehmen Konflikten [30, 31], die eigene Betroffenheit [24] sowie keine gemeinsam vereinbarten Richtlinien [29] überfordern medizinisches Fachpersonal, verhindern die entsprechende Diagnose und führen dazu, dass möglicherweise begründete (Verdachts-)Fälle von Kindeswohlgefährdung übersehen, verdrängt und/oder keiner fachlich angemessenen Prüfung zugeführt und unterzogen werden. Die hohe Rate an bestätigten Misshandlungen im ers- 


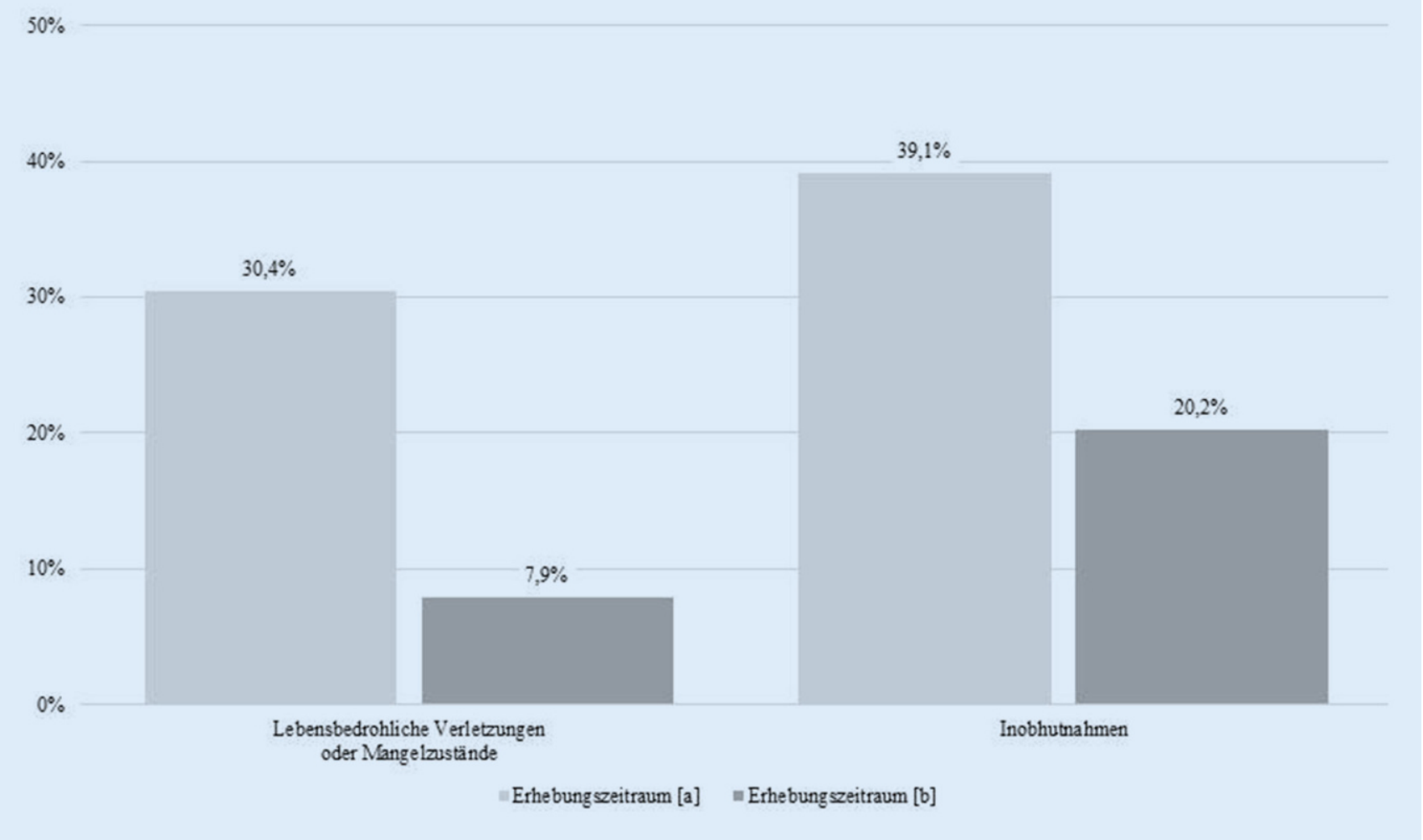

Abb. 4 L Lebensbedrohliche Verletzungen oder Mangelzustände $(n=14)$ und Inobhutnahmen $(n=27)$ der Erhebungszeiträume [a] und [b]

ten Erhebungszeitraum (95,5\%) spricht vermutlich dafür, dass nur die schwerwiegendsten Fälle wahrgenommen und diagnostiziert wurden.

Mit der Implementierung der hauptamtlichen Kinderschutzkoordinatorin wurde versucht, derartigen Mechanismen effektiv entgegenzuwirken. Zusätzliche berufsgruppenspezifische Fortbildungsmaßnahmen forcierten die Sensibilisierung, das Bewusstsein spezifischer gesetzlicher Bestimmungen sowie die daraus resultierende berufsrechtliche und nicht zuletzt berufsethische Verantwortung von medizinischen Fachkräften. Damit erhielt der Kinderschutz im beruflichen Alltag eine erhöhte Aufmerksamkeit und Wahrnehmung. Auf der Basis einer erheblich gestiegenen Bereitschaft zu interdisziplinärem Handeln konnten (Verdachts-)Fälle von Kindeswohlgefährdung unkompliziert und niedrigschwellig gemeldet und unter der Leitung und Steuerung der hauptamtlichen Kinderschutzkoordinatorin dem bewährten multiprofessionellen Abklärungsprozess unterzogen werden, während die im klinischen Alltag ohnehin stark beanspruchten personellen Ressourcen geschont wurden. Damit konnte der Ressourcenknappheit, die in der Literatur als mitursächlich für das Übersehen von Kindeswohlgefährdung angesehen wird, erfolgreich entgegengewirkt werden [30-32]. Die Fallsteuerung sowie die gesamte Kommunikation wurden von der hauptamtlichen Kinderschutzkoordinatorin übernommen und waren dadurch nicht mehr von den behandelnden Ärzten im meist schon sehr ausgefüllten Klinikalltag zu leisten.

Auf der Grundlage des mittlerweile erlöswirksamen OPS sowie der Empfehlungen der DGKiM [24] wurden verbindliche Verfahrensabläufe und Standards entwickelt, die der individuellen örtlichen Struktur und den personellen Gegebenheiten angepasst worden waren. Sie vermitteln dem Klinikpersonal insbesondere in akuten Situationen Sicherheit, gewährleisten ein strukturiertes Vorgehen und vermeiden überstürztes Handeln oder Einzelaktivitäten.
Der immense Anstieg der Prävalenz der Fälle, bei denen sich im Rahmen des Abklärungsprozesses der Verdacht auf Kindeswohlgefährdung erhärtet hat, zeigt den dringenden Bedarf sowie das Potenzial zusätzlicher personeller Ressourcen, Kompetenzen und Verantwortlichkeiten im medizinischen Kinderschutz. Das Modellprojekt am Altonaer Kinderkrankenhaus scheint wirksam, um erwähnten Mechanismen zu begegnen, (Verdachts-)Fälle von Kindeswohlgefährdung zuverlässiger zu erkennen und abzuklären und damit die erhebliche Dunkelziffer in diesem Bereich zu reduzieren.

Die signifikante Abnahme der Kinderschutzfälle mit festgestellten lebensbedrohlichen Verletzungen oder Mangelerscheinungen erlaubt den Rückschluss, dass eine mutmaßliche Reduzierung der Dunkelziffer in besonderem Maße bei solchen (Verdachts-)Fällen wirksam geworden ist, bei denen die Kinder und Jugendlichen weniger gravierende Anzeichen von Kindeswohlgefährdung aufgewiesen haben, damit schwieriger zu er- 


\begin{tabular}{lll}
\hline \begin{tabular}{ll} 
Tab. 1 Hinweise auf Kindeswohlgefährdung der Erhebungszeiträume [a] und [b] \\
\hline Diagnostische und anamnestische Auffälligkeiten
\end{tabular} & $\begin{array}{l}\text { Erhebungs- } \\
\text { zeitraum [a] } \\
\text { (in \%) }\end{array}$ & $\begin{array}{l}\text { Erhebungs- } \\
\text { zeitraum [b] } \\
\text { (in \%) }\end{array}$ \\
\hline $\begin{array}{l}\text { Zeitlich verzögerte Vorstellung } \\
\text { Fehlende, unzureichende und wechselnde Erklärungen }\end{array}$ & 58,3 & 40,5 \\
\hline $\begin{array}{l}\text { Inadäquater Unfallmechanismus bzw. Entwicklungsstand } \\
\text { Angebliche Verursachung durch Geschwisterkind }\end{array}$ & 41,7 & 35,1 \\
\hline $\begin{array}{l}\text { Hinweis von Kind selbst } \\
\text { Weitere Verletzungen ohne Bezug zum primären Vorstellungs- } \\
\text { grund }\end{array}$ & 25,0 & 2,7 \\
\hline $\begin{array}{l}\text { Unklare, rezidivierende Verletzungen } \\
\text { Überweisung „V.a. Kindesmisshandlung“ }\end{array}$ & 33,3 & 13,5 \\
\hline
\end{tabular}

kennen waren und in der Vergangenheit wahrscheinlich eher unerkannt blieben. Es erscheint eingängig, dass gravierendere und eindeutigere (Verdachts-)Fälle mit weniger Unsicherheiten und Zurückhaltung einhergehen sowie Hemmnisse und Hürden bei der Verfolgung eines Verdachts weniger ausgeprägt sind. Hier scheint sich ein Abklärungsbedürfnis vehementer durchzusetzen. Die Schlussfolgerung, dass schwere Verletzungen häufiger gemeldet werden, deckt sich mit dem Ergebnis einer internationalen Studie [33]. Nach der Neustrukturierung konnten (Verdachts-)Fälle, die weniger offensichtlich imponierten, ebenfalls erkannt und einer fachlichen Prüfung unterzogen werden. Auch das Ergebnis, dass die einfacher zu erhebenden Diagnose- und Anamneseauffälligkeiten im Untersuchungszeitraum [a] deutlich höher repräsentiert sind, bestärkt die Annahme, dass mit der Neukonzeptionierung des Kinderschutzes im Zeitraum [b] auch weniger offensichtliche (Verdachts-)Fälle abgeklärt werden konnten. Vor dem Hintergrund, dass Kindeswohlgefährdung kein einmaliges Ereignis darstellt und von einer stets bestehenden Möglichkeit zunehmend schwerer Verletzungen auszugehen ist [34-37], könnte das frühzeitige Erkennen von geringfügigeren Anzeichen einer potenziellen Eskalation zuvorkommen und einen potenziellen Leidensweg der Kinder verkürzen sowie weitere Folgen mindern.

Die Untersuchung zeigt allerdings auch, dass mit der Implementierung der hauptamtlichen Kinderschutzkoordinatorin die Prävalenz der falsch-positiven
Verdachtsfälle signifikant gestiegen ist. Das etablierte Vorgehen des Altonaer Kinderkrankenhauses stellt allerdings einen überaus sensiblen Umgang mit Verdachtsäußerungen sicher, um die familiäre Integrität bestmöglich zu schützen. Die Konfrontation der Eltern und damit das Äußern des Verdachts sowie das Einleiten weiterer Maßnahmen erfolgen erst und ausschließlich nach ausführlicher Beratung und Auswertung der Befunde durch die multiprofessionelle Kinderschutzgruppe, sofern von der Erhärtung des Verdachts auszugehen ist. Dieses Vorgehen ist essenziell, um Fehlinterpretationen zu minimieren und unzutreffende Verdachtsäußerungen sowie Stigmatisierungen zu vermeiden. Positiv festzuhalten ist, dass die Prävalenz der erhärteten Kinderschutzfälle hoch signifikant gestiegen ist, also im Zuge des Anstiegs der überprüften (Verdachts-)Fälle eine vielfach höhere Zahl von erhärteten Kinderschutzfällen festgestellt werden konnte.

Die Regionen der körperlichen Verletzungen unterscheiden sich in beiden Gruppen nicht. So ist in der Gesamtheit der Fälle beider Untersuchungszeiträume die vulnerable Kopf-Hals-Region führend, jedoch zeigt die Verteilung auf alle Körperregionen, dass eine ausgiebige körperliche Untersuchung obligat ist. Neben Weichteilverletzungen $(55,0 \%)$ treten Frakturen mit 24,5\% am zweithäufigsten auf. Bei der Beurteilung kindlicher Frakturen ist neben der alterstypischen Frakturverteilung auch insbesondere auf den Entwicklungsstand des Kindes und die davon beeinflusste Plausibilität des beschriebenen Unfallmechanis- mus zu achten [38]. Thermische Verletzungen treten mit 2,0\% verhältnismäBig selten auf. In einer retrospektiven Untersuchung brandverletzter Kinder in Deutschland wurde aufgezeigt, dass eine große Differenz zu vergleichbaren internationalen Populationen vorliegt und damit eine große Dunkelziffer vorzuliegen scheint [39].

\section{Limitationen}

Die vorliegende Studie ist eine retrospektive Untersuchung, die anhand der elektronischen Patientenakten eine umfassende Aussage über die untersuchten Erhebungszeiträume zu treffen versucht. Alle erfassten (Verdachts-)Fälle sind in den Patientenakten eindeutig gekennzeichnet, was die Abfrage vereinfacht und eine vollständige Abbildung der Fallzahlen gesichert hat. Teile der erhobenen Parameter wurden aus den dokumentierten Anamnesen extrahiert, womit die Vollständigkeit nicht sicher gewährleistet werden kann. Die Anamneseerhebung erfolgte jedoch, wie beim Verdacht auf Kindeswohlgefährdung obligat, ausführlich und penibel, wodurch eine Reduktion der Fehlerquote angenommen werden kann.

Nicht auszuschließen ist der Einfluss zunehmender Expertise bei den Mitgliedern der Kinderschutzgruppe sowie kontinuierlicher Weiterentwicklungen im medizinischen Kinderschutz auf die Qualität der klinischen Kinderschutzarbeit in den unterschiedlichen Erhebungszeiträumen. Dieser Einfluss konnte jedoch nicht definiert oder statistisch berücksichtigt werden. Ebenso wenig in ihrer Auswirkung auf die gestiegene Zahl der Diagnosen zu erfassen und zu bewerten sind die am Anfang der Diskussion beschriebenen allgemeinen gesellschaftlichen (Bundeskinderschutzgesetz, mehr mediale Präsenz etc.) und fachpolitischen Entwicklungen und die Etablierung des medizinischen Kinderschutzes als eigenes Fachgebiet in der letzten Dekade (bessere Ausbildung, mehr Fachveröffentlichungen und Standards, Präsenz auf Kongressen etc.). 


\section{Resümee}

Das vorgestellte Konzept zum medizinischen Kinderschutz in Kinderkliniken scheint das Hellfeld bei Kindeswohlgefährdung effektiv auszuweiten. Der deutliche Prävalenzanstieg sowie die verringerte Anzahl der lebensbedrohlichen Verletzungen bei vergleichbaren Populationen der Untersuchungszeiträume stellen Hinweise auf eine Verringerung der Dunkelziffer dar. Die personelle Investition ist einerseits durch die zwischenzeitlich erlöswirksame OPS zu rechtfertigen, andererseits insbesondere auch durch die Reduktion der vermeidbaren immensen gesellschaftlichen Folgekosten. Darüber hinaus sollte jedoch die Verhinderung von potenziellen Eskalationen sowie potenziellen Leidenswegen der Kinder genügend Anreiz für eine wie dargestellt qualitativ hochwertige Kinderschutzarbeit sein.

\section{Korrespondenzadresse}

\section{Dr. med. Ingo Königs}

Abteilung für Kinderchirurgie, Altonaer Kinderkrankenhaus

Bleickenallee 38, 22763 Hamburg, Deutschland ingo.koenigs@uke.de

Funding. Open Access funding provided by Projekt DEAL.

\section{Einhaltung ethischer Richtlinien}

Interessenkonflikt. C.M. Schmidt, S. Klein, P. Stock, K. Reinshagen und I. Königs geben an, dass kein Interessenkonflikt besteht.

Für diesen Beitrag wurden von den Autoren keine Studien an Menschen oder Tieren durchgeführt. Für die aufgeführten Studien gelten die jeweils dort angegebenen ethischen Richtlinien.

Open Access. Dieser Artikel wird unter der Creative Commons Namensnennung 4.0 International Lizenz veröffentlicht, welche die Nutzung, Vervielfältigung, Bearbeitung, Verbreitung und Wiedergabe in jeglichem Medium und Format erlaubt, sofern Sie den/die ursprünglichen Autor(en) und die Quelle ordnungsgemäß nennen, einen Link zur Creative Commons Lizenz beifügen und angeben, ob Änderungen vorgenommen wurden.

Die in diesem Artikel enthaltenen Bilder und sonstiges Drittmaterial unterliegen ebenfalls der genannten Creative Commons Lizenz, sofern sich aus der Abbildungslegende nichts anderes ergibt. Sofern das betreffende Material nicht unter der genannten Creative Commons Lizenz steht und die betreffende Handlung nicht nach gesetzlichen Vorschriften erlaubt ist, ist für die oben aufgeführten Weiterverwendungen des Materials die Einwilligung des jeweiligen Rechteinhabers einzuholen.

Weitere Details zur Lizenz entnehmen Sie bitte der Lizenzinformation auf http://creativecommons.org/ licenses/by/4.0/deed.de.

\section{Literatur}

1. Fendrich S, Pothmann J (2010) Einblicke in die Datenlage zur Kindesvernachlässigung und Kindesmisshandlung in Deutschland. Möglichkeiten und Grenzen von Gesundheits-, Kriminal- und Sozialstatistiken. Bundesgesundheitsblatt Gesundheitsforschung Gesundheitsschutz 53:1002-1010

2. AlEissa MA, Fluke JD, Gerbaka B et al (2009) A commentary on national child maltreatment surveillance systems: Examples of progress. Child Abuse Negl 33:809-814

3. Statistisches Bundesamt (2020) Gefährdungseinschätzungen nach § 8a Absatz 1 SGB VII 2017. https://www.destatis.de/DE/Themen/ Gesellschaft-Umwelt/Soziales/KinderhilfeJugendhilfe/Publikationen/Downloads-Kinderund-Jugendhilfe/gefaehrdungseinschaetzungen5225123177004.pdf?_blob=publicationFile\& v=4.Zugegriffen: 20 . März 2020

4. Bundesärztekammer (2020) Vernachlässigung und Misshandlung von Kindern: Früherkennung und Prävention als ärztliche Aufgabe. https://www.bundesaerztekammer.de/aerzte/ versorgung/praevention/kindesmisshandlung/. Zugegriffen: 20. März 2020

5. Pillhofer M, Ziegenhain U, Nandi C, Fegert JM, Goldbeck L (2011) Prävalenz von Kindesmisshandlung und -vernachlässigung in Deutschland. Annäherung an ein Dunkelfeld. Kindh Entwickl 20:64-71

6. Häuser W, Schmutzer G, Brähler E, Glaesmer H (2011) Misshandlungen in Kindheit und Jugend. Dtsch Arztebl 108:287-294

7. Stoltenborgh M, Bakermans-Kranenburg MJ, AlinkLR, van IJzendoorn MH (2015) The prevalence of child maltreatment across the globe: review of a series of meta-analyses. Child Abus Rev 24(1):37-50

8. Finkelhor D (1994) The international epidemiology of child sexual abuse. Child Abuse Negl 18:409-417

9. Finkelhor D, Turner $H$, Ormrod R, Hamby SL (2009) Violence, abuse, and crime exposure in a national sample of children and youth. Pediatrics 124:1411-1423

10. Pereda N, Guilera G, Forns M, Gomez-Benito J (2009) The international epidemiology of child sexual abuse: a continuation of Finkelhor. Child Abuse Negl 33:331-342

11. Maguire SA, Williams B, Naughton AM et al (2015) A systematic review of the emotional, behavioural and cognitive features exhibited by school-aged children experiencing neglect or emotional abuse. Child Care Health Dev 41:641-653

12. Widom CS, Czaja SJ, Bentley T, Johnson MS (2012) A prospective investigation of physical health outcomes in abused and neglected children: new findings from a 30-year follow-up. Am J Public Health Res 102:1135-1144

13. Krug EG, Dahlberg LL, Mercy JA, Zwi AB, Lozano RL (2020) World report on violence and health 2002. https://apps.who.int/iris/ bitstream/handle/10665/42495/9241545615 eng.pdf? sequence $=1$.Zugegriffen: 20. März 2020

14. Heim C, Shugart M, Craighead WE, Nemeroff CB (2010) Neurobiological and psychiatric consequences of child abuse and neglect. Dev Psychobiol 52:671-690

15. Norman RE, Byambaa M, De R, Butchart A, Scott J, Vos $T$ (2012) The long-term health consequences of child physical abuse, emotional abuse, and neglect: a systematic review and meta-analysis. PLoS Med 9:e1001349

16. Pechtel P, Pizzagalli DA (2011) Effects of early life stress on cognitive and affective function: an integrated review of human literature. Psychopharmacology 214:55-70

17. Perez CM, Widom CS (1994) Childhood victimization and longterm intellectual and academic outcomes. Child Abuse Negl 18:617-633

18. Jonson-Reid M, Drake B, Kim J, Porterfield S, Han L (2004) A prospective analysis of the relationship between reported child maltreatment and special education eligibility among poor children. Child Maltreat 9:382-394

19. Herrenkohl TI, Herrenkohl RC (2007) Examining the overlap and prediction of multiple forms of child maltreatment, stressors, and socioeconomic status: a longitudinal analysis of youth outcomes. JFam Violence 22:553-562

20. Sternberg KJ, Lamb ME, Guterman E, Abbott CB (2006) Effects of early and later family violence on children's behavior problems and depression: a longitudinal, multi-informant perspective. Child Abuse Negl 30:283-306

21. Lansford JE, Miller-Johnson S, Berlin LJ, Dodge KA, Bates JE, Pettit GS (2007) Early physical abuse and later violent delinquency: a prospective longitudinal study. Child Maltreat 12:233-245

22. Stouthamer-Loeber M, Loeber R, Homish DL, Wei E (2001) Maltreatment of boys and the development of disruptive and delinquent behavior. Dev Psychopathol 13:941-955

23. Habetha S, Bleich S, Weidenhammer J, Fegert JM (2012) A prevalence-based approach to societal costs occurring in consequence of child abuse and neglect. Child Adolesc Psychiatry Ment Health 6:35

24. Deutsche Akademie für Kinder- und Jugendmedizin e.V. (DAKJ), Deutsche Gesellschaft für Kinderschutz in der Medizin (DGKiM) (2016) Deutsche AkademiefürKinder-und Jugendmedizine. V. (DAKJ) und DeutscheGesellschaftfür Kinderschutz in der Medizin (DGKiM): Vorgehen bei Kindesmisshandlung und -vernachlässigung. Empfehlungen für Kinderschutz an Kliniken. Version 1.6, 11/2016. https://www.dgkim.de/dateien/empfehlungenkinderschutz-an-kliniken-2016.pdf. Zugegriffen: 20. März 2020

25. Kinderschutzleitlinienbüro (2018) AWMF S3+ Leitlinie Kindesmisshandlung, -missbrauch, -vernachlässigung unter Einbindung der Jugendhilfe und Pädagogik [Kinderschutzleitlinie], Langfassung 1.0, 2018, AWMF-Registernummer: 027-069. https://www.awmf.org/uploads/tx_szleitlinien/ KF_027-069k_Kinderschutz_2018-10.pdf. Zugegriffen:20. März2020

26. Universitätsklinikum UIm (2020) E-Learning Kinderschutz. https://elearning-kinderschutz.de. Zugegriffen: 20. März 2020

27. Universitätsklinikum UIm (2020) Medizinische Kinderschutzhotline. https://www. kinderschutzhotline.de. Zugegriffen: 20. März 2020

28. Cui N, Xue J, Connolly CA, Liu J (2016) Does the gender of parent or child matter in child maltreatment in China? Child Abuse Negl 54:1-9 
29. Paavilainen $E$, Astedt-Kurki P, Paunonen-Ilmonen M, Laippala P (2002) Caring for maltreated children: a challenge for health care education. JAdv Nurs 37:551-557

30. Tiyyagura G, Gawel M, Koziel JR, Asnes A, Bechtel K (2015) Barriers and facilitators to detecting child abuse and neglect in general emergency departments. Ann Emerg Med 66:447-454

31. Louwers EC, Korfage IJ, Affourtit MJ, De Koning HJ, MollHA(2012) Facilitators and barriers to screening for child abuse in the emergency department. BMC Pediatr 12:167

32. Socolar RR, Reives P (2002) Factors that facilitate or impede physicians who perform evaluations for child maltreatment. Child Maltreat 7:377-381

33. Flaherty EG, Sege RD, Griffith J et al (2008) From suspicion of physical child abuse to reporting: primary care clinician decision-making. Pediatrics 122:611-619

34. King WK, Kiesel EL, Simon HK (2006) Child abuse fatalities: are we missing opportunities for intervention? Pediatr Emerg Care 22:211-214

35. Oral R, Yagmur F, Nashelsky M, Turkmen M, Kirby P (2008) Fatal abusive head trauma cases: consequence of medical staffmissing milder forms of physical abuse. Pediatr Emerg Care 24:816-821

36. Alexander R, Crabbe L, Sato Y, Smith W, Bennett T (1990) Serial abuse in children who are shaken. Am J Dis Child 144:58-60

37. Ravichandiran N, Schuh S, Bejuk M et al (2010) Delayed identification of pediatric abuse-related fractures. Pediatrics 125:60-66

38. Berthold O, Frericks B, John T, Clemens V, Fegert JM, Moers AV (2018) Abuse as a cause of childhood fractures. Dtsch Arztebl Int 115:769-775

39. Klinke M, Schmidt CM, Tegtmeyer L, Reinshagen K, Boettcher M, Koenigs I (2018) Undetected cases of non-accidental burns in children-preventive strategies. Klin Padiatr 230:61-67 